\title{
THE INVERSE STATIONARY HEAT CONDUCTION PROBLEM FOR A CUBOID
}

\author{
Konstantin M.Yacenko ${ }^{1,}$, Yri Y.Rakov ${ }^{1}$, and Konstantin V. Slyusarskiy ${ }^{1}$ \\ ${ }^{1}$ Tomsk Polytechnic University, 634050, Tomsk, Russia
}

\begin{abstract}
The heat conductivity coefficient is important characteristic which is used in various spheres. The original methodic for conductivity coefficient determination was proposed for samples in form of rectangular parallelepiped. The results of numerical solution of nonlinear heat conduction problem in heat conduction coefficient value range $0.04-5$ $\mathrm{W} /(\mathrm{m} \mathrm{K})$ with different sample relative size were presented.
\end{abstract}

\section{Introduction}

The heat conduction of substance is widespread phenomena and has a strong influence on various aspects of technological processes: from durability calculations to heat flux determination [1]. The heat conduction coefficient determines substance behavior in terms of heat conduction and extremely important for its correct description [2]. Existing experimental procedures for its estimation often have to handle with heat-transfer coefficient which is hard to measure directly. This causes significant error in heat conductivity coefficient final values [3]. To eliminate influence of this factor on measurements this procedure is realized in vacuum. To estimate exploitation parameters for measuring procedure for substances with heat conductivity value in range $0.04-5 \mathrm{~W} /(\mathrm{m} \mathrm{K})$ the physical model of heat transfer was formulated and investigated.

\section{Physical model of heat transfer}

The test object is a model of a cuboid with the dimensions LX, LY, LZ. It is heated by the heat flux at one side but loses heat from all sides to the environment by radiation. Ambient temperature is constant and equal to $T_{o c}$. Integral emission coefficient $\varepsilon$ and absorption coefficient of the sample surface $A$ will take constant and coefficient of thermal conductivity $\lambda$ does not depend on temperature [4].

\footnotetext{
${ }^{*}$ Corresponding author: brkmax@mail.ru
} 


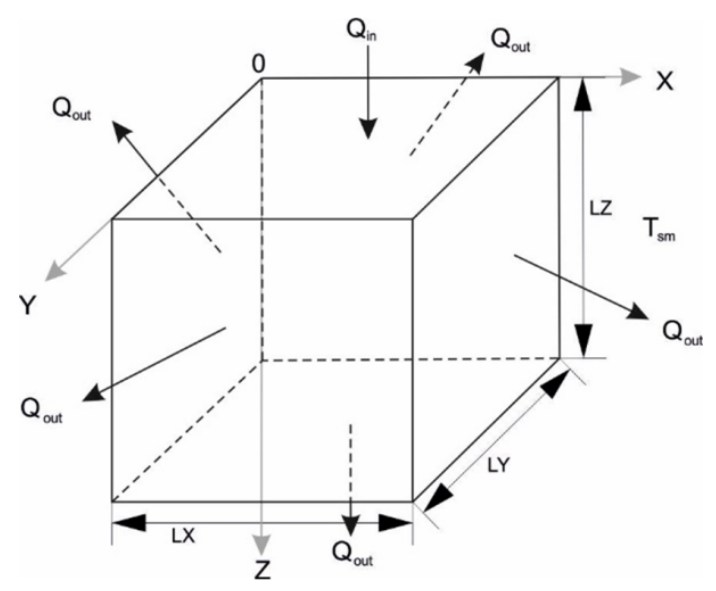

Fig. 1. The scheme of distribution of heat fluxes in the sample.

\section{Mathematical model and decision methods}

The mathematical dimensionless statement of the inverse stationary heat conduction problem for cuboid includes the heat equation (1), non-linear boundary conditions (2 - 7) and extreme conditions (8):

$$
\begin{gathered}
\frac{\partial^{2} \tilde{T}}{\partial \tilde{x}^{2}}+\frac{\partial^{2} \tilde{T}}{\partial \tilde{y}^{2}}+\frac{\partial^{2} \tilde{T}}{\partial \tilde{z}^{2}}=0 \\
\left(\frac{\partial \tilde{T}}{\partial \tilde{z}}\right)_{\tilde{z}=0}=\pi_{1} \cdot\left(\tilde{T}_{\tilde{z}=0}^{4}-\tilde{T}_{o c}^{4}\right)-1 \\
-\left(\frac{\partial \tilde{T}}{\partial \tilde{z}}\right)_{\tilde{z}=1}=\pi_{1} \cdot\left(\tilde{T}_{\tilde{z}=1}^{4}-\tilde{T}_{o c}^{4}\right) \\
\left(\frac{\partial \tilde{T}}{\partial \tilde{x}}\right)_{\pi_{2}=0}=\pi_{1} \cdot\left(\tilde{T}_{\tilde{x}=0}^{4}-\tilde{T}_{o c}^{4}\right) \\
-\left(\frac{\partial \tilde{T}}{\partial \tilde{x}}\right)_{\pi_{2}}=\pi_{1} \cdot\left(\tilde{T}_{\pi_{2}}^{4}-\tilde{T}_{o c}^{4}\right) \\
\left(\frac{\partial \tilde{T}}{\partial \tilde{y}}\right)_{\pi_{3}=0}=\pi_{1} \cdot\left(\tilde{T}_{\tilde{y}=0}^{4}-\tilde{T}_{o c}^{4}\right) \\
-\left(\frac{\partial \tilde{T}}{\partial \tilde{y}}\right)_{\pi_{3}}=\pi_{1} \cdot\left(\tilde{T}_{\pi_{3}}^{4}-\tilde{T}_{o c}^{4}\right) \\
\pi_{4}-\int_{\tilde{F}}\left[\tilde{T}_{\tilde{z}=1}^{4}-\tilde{T}_{o c}^{4}\right] \partial \tilde{F} \mid \rightarrow \min
\end{gathered}
$$


Here $\pi_{1}=\frac{\varepsilon \sigma \cdot L Z^{4} \cdot q_{F}^{3} A^{3}}{\lambda^{4}}, \pi_{2}=\frac{L X}{L Z}, \pi_{3}=\frac{L Y}{L Z}, \pi_{4}=\frac{Q_{L Z} \lambda^{4}}{\varepsilon \sigma q_{F}^{4} A^{4} \cdot L Z^{6}} ; \tilde{T}=T /\left(\frac{q_{F} A \cdot L Z}{\lambda}\right)$, $\tilde{T}_{o c}=T_{o c} /\left(\frac{q_{F} A \cdot L Z}{\lambda}\right), \tilde{F}=\frac{F}{L Z^{2}}, \tilde{x}=\frac{X}{L Z}, \tilde{y}=\frac{Y}{L Z}, \tilde{z}=\frac{Z}{L Z} ; Q_{L Z}$ is integral heat flux from the bottom surface of the sample.

As follows from the mathematical statement of the inverse problem, the solution of the direct problem of heat conduction in different numerical values of coefficient of the thermal conductivity is necessary to calculate the minimum of objective function (2.8).

Numerical methods are used to solve nonlinear problems to get a table of approximate values. The finite difference method was chosen for this task.

Finite difference equations were solved by a method of fixed-point iteration and iterative method of successive over-relaxation (accelerated Liebmann method). The dichotomy method was used to determine the coefficient of thermal conductivity in the inverse problem [5].

\section{Results and discussion}

Figure 2 shows the dependence between the dimensionless parameters $\pi_{1}, \pi_{2}, \pi_{3}, \pi_{4}$ for the estimation of the geometric dimensions of the sample and the physical parameters of the experiment. It is necessary to determine the coefficient of thermal conductivity in the estimated range of its values.

The value $\pi_{1}$ should be in the range of $0.001-0.01$ when the relative sizes of the sample $\pi_{2}=\pi_{3}$ corresponding to the range from to 1 to 10 . In this case, temperature changes in the sample will not exceed $100 \mathrm{~K}$ and use of mathematical models (1-8) becomes possible.

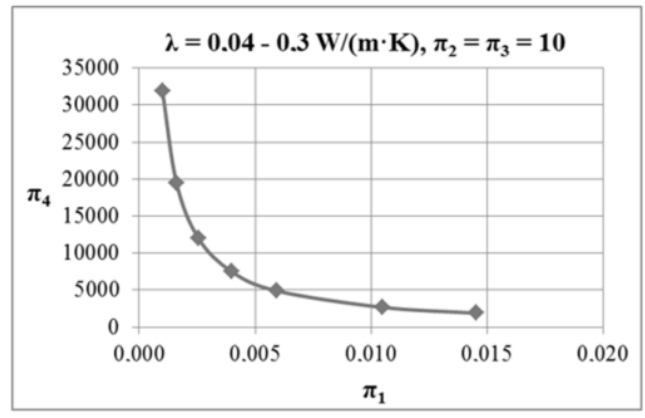

a

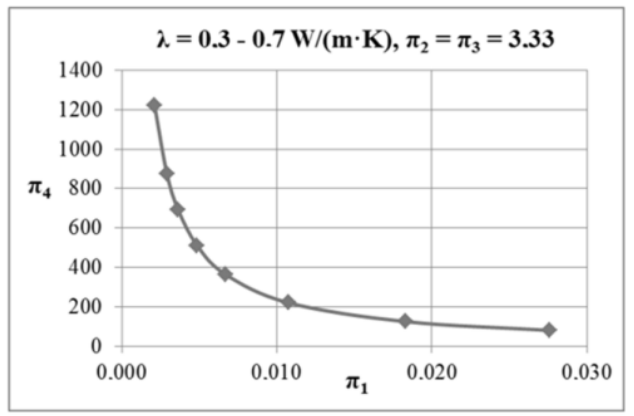

b 

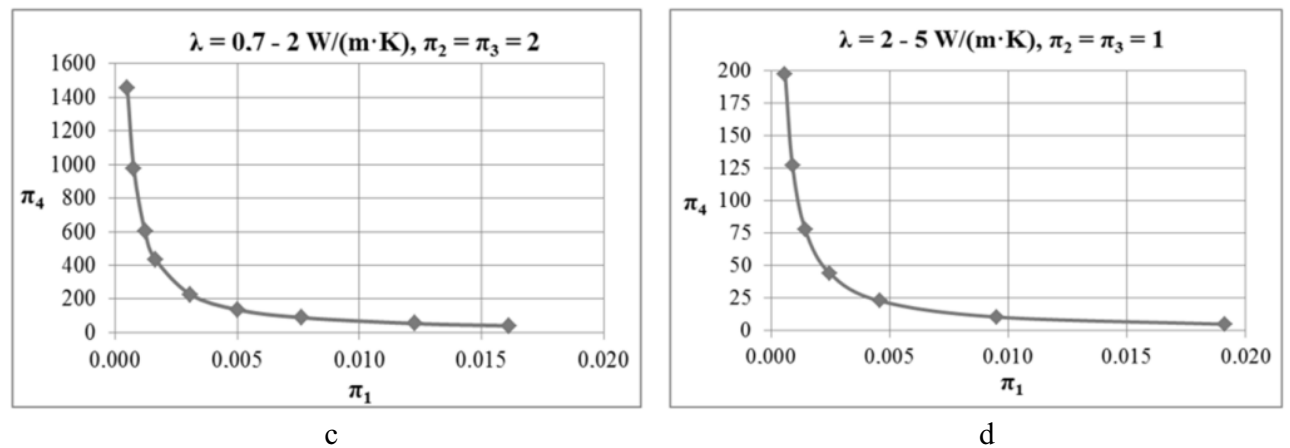

Fig. 2. The dependence between $\pi_{4}$ and $\pi_{1}: \mathrm{a}-\lambda=0.04-0.3 \mathrm{~W} /(\mathrm{m} \cdot \mathrm{K}), \pi_{2}=\pi_{3}=10 ; \mathrm{b}-\lambda=0.3-0.7$ $\mathrm{W} /(\mathrm{m} \cdot \mathrm{K}), \pi_{2}=\pi_{3}=3.33 ; \mathrm{c}-\lambda=0.7-2 \mathrm{~W} /(\mathrm{m} \cdot \mathrm{K}), \pi_{2}=\pi_{3}=2 ; \mathrm{a}-\lambda=2-5 \mathrm{~W} /(\mathrm{m} \cdot \mathrm{K}), \pi_{2}=\pi_{3}=1$.

\section{Conclusion}

The physical and mathematical model for thermal conductivity coefficient determination was formed for cubic sample. The optimal parameters for sample size and thermal flows were calculated in $\lambda$ range $0.04-5 \mathrm{~W} /(\mathrm{m} \mathrm{K})$.

This work was supported by Russian Science Foundation (project No. 16-19-10316).

\section{References}

1. K.V. Slyusarskiy, M.K. Bejsekov, J.V. Marysheva, Y.J. Rakov, MATEC Web Conf. 37, 01053 (2015)

2. T.E. Shoup, Applied numerical methods for microcomputers (Higher School, Moskow, 1984)

3. E.P. Mikhalev, Yu. Ya. Rakov, 9 Int Sci Pract Conf Stud Post-Grad Young Sci. 1, 56 (2003)

4. A.S. Zavorin, A.V. Kuzmin, Y.Y. Rakov, Methods for determination the condensed matter thermal conductivity: tutorial (Publishing house TPU, Tomsk, 2009)

5. D.D. McCracken, W.S. Dorn, Numerical methods and Fortran programming with application in engineering and science (Business Machines Corporation: Wiley international edition, 1965) 